\title{
SUMS OF CLASS NUMBERS AND MIXED MOCK MODULAR FORMS
}

\author{
KATHRIN BRINGMANN AND BEN KANE
}

\begin{abstract}
In this paper, we consider sums of class numbers of the type $\sum_{m \equiv a(\bmod p)} H\left(4 n-m^{2}\right)$, where $p$ is an odd prime, $n \in \mathbb{N}$, and $a \in \mathbb{Z}$. By showing that these are coefficients of mixed mock modular forms, we obtain explicit formulas. Using these formulas for $p=5$ and 7 , we then prove a conjecture of Brown et al. in the case that $n=\ell$ is prime.
\end{abstract}

\section{IntRoduction AND STATEMENTS OF RESUlts}

Let $H(n)$ denote the $n$th Hurwitz class number, i.e., the number of equivalence classes of positive definite binary quadratic forms of discriminant $-n$ with the class containing $x^{2}+y^{2}$ weighted by $\frac{1}{2}$ and the class containing $x^{2}+x y+y^{2}$ weighted by $\frac{1}{3}$. Moreover, by convention $H(0)=-\frac{1}{12}$. Certain congruence classes appear as coefficients of weight $\frac{3}{2}$ Eisenstein series. However, the generating function for all Hurwitz class numbers

$$
\mathcal{H}(q):=\sum_{n \geq 0} H(n) q^{n}
$$

is not itself modular, but rather mock modular 9]. Roughly speaking, this means that $\mathcal{H}$ may be naturally "completed" to a non-holomorphic modular form (a further description is given in Section (2). Mock modular forms have since shown up in a variety of applications. To name a few examples, Ramanujan's mock theta functions have been shown to be mock modular forms [19], they have led to asymptotic and exact formulas in partition theory [3, 5, they are related to Lie superalgebras [1, 4, 15], and they are connected to the quantum theory of black holes [2, 7].

Date: November 2, 2018.

2010 Mathematics Subject Classification. 11E41, 11F37, 11F30.

Key words and phrases. class numbers, Lerch sums, mixed mock modular forms, mock theta functions, modular forms.

The research of the first author was supported by the Alfried Krupp Prize for Young University Teachers of the Krupp Foundation. This research was completed while the second author was a postdoc at the University of Cologne. 
In this paper, we prove conjectures reminiscent of the famous identity (cf. p. 154 of [8])

$$
\sum_{|m|<2 \sqrt{\ell}} H\left(4 \ell-m^{2}\right)=2 \ell,
$$

where $\ell$ is an odd prime. More specifically, for a prime $p, a \in \mathbb{Z}$, and $n \in \mathbb{N}$, this paper is focused on sums of the type

$$
H_{a, p}(n):=\sum_{\substack{|m| \leq 2 \sqrt{n} \\ m \equiv a}} H\left(4 n-m^{2}\right) .
$$

A number of identities for $H_{a, p}(n)$ were obtained in [6] for the special cases that $n=\ell$ is prime and $p=2,3,5$, or 7 . To give an example indicative of the results in [6], they proved in the special case that $p=5$ and $n=\ell$ is prime that

$$
H_{a, 5}(\ell)= \begin{cases}\frac{\ell-3}{2} & \text { if } a \equiv 0 \quad(\bmod 5), \text { and } \ell \equiv 4 \quad(\bmod 5) \\ \frac{\ell-1}{2} & \text { if } a \equiv \pm 1 \quad(\bmod 5), \text { and } \ell \equiv 3 \quad(\bmod 5) \\ \frac{\ell-1}{2} & \text { if } a \equiv \pm 2 \quad(\bmod 5), \text { and } \ell \equiv 2 \quad(\bmod 5)\end{cases}
$$

In the cases $p=5$ and $p=7$, they were unable to completely classify $H_{a, p}(\ell)$, but conjecture a number of pleasant identities similar to (1.2) based on computer data.

Conjecture 1.1. For every $a, L \in \mathbb{Z}$, there exist constants $c_{1}, c_{2} \in \mathbb{Q}$ (given explicity in (4.3)) such that for every prime $\ell \equiv L(\bmod 5)$, we have

$$
H_{a, 5}(\ell)=c_{1} \ell+c_{2} \text {. }
$$

When restricted to certain congruence classes for $a$ and $\ell(\bmod 7)$, they conjecture a similar formula for $H_{a, 7}(\ell)$.

Conjecture 1.2. If $L=3,5,6$ and $a \in \mathbb{Z}$ or $(a, L) \equiv( \pm 1,1)(\bmod 7)$, then there exist constants $c_{1}, c_{2} \in \mathbb{Q}$ (given explicitly in (4.4) ) such that for every prime $\ell \equiv L$ $(\bmod 7)$, we have

$$
H_{a, 7}(\ell)=c_{1} \ell+c_{2} .
$$

We settle these conjectures in this paper.

Theorem 1.3. Conjectures 1.1 and 1.2 are true.

Theorem 1.3 is implied by a more general theorem, which we next describe. The key ingredient is to use the mock modularity of the Hurwitz class number generating function, whereas the authors of [6] only took advantage of the modularity of certain congruence classes. In particular, $H_{a, p}(n)$ are the coefficients of what are known as 
mixed mock modular forms, which are products of mock modular forms with modular forms.

We construct explicit mixed mock modular forms with these coefficients from the product of $\mathcal{H}(q)$ with unary theta functions $\vartheta_{a, N}(\tau)$ defined in (3.1) (where $N \in \mathbb{N}$ and $q:=e^{2 \pi i \tau}$ throughout). To completely describe these, for a series $f(\tau)=\sum_{n \in \mathbb{Z}} a(n) q^{n}$, we require the $d$ th $U$-operator $f \mid U(d)(\tau):=\sum_{n \in \mathbb{Z}} a(n d) q^{n}$ and the twist of a series $f$ by a character $\chi$, i.e., $f \otimes \chi(\tau):=\sum_{n \in \mathbb{Z}} a(n) \chi(n) q^{n}$. It is straightforward to see that for every $a \in \mathbb{Z}$ and prime $p$, one has

$$
\sum_{\substack{n \geq 0 \\ p \nmid n}} H_{a, p}(n) q^{n}=\left(\mathcal{H}(q) \vartheta_{a, p}(\tau)\right) \mid U(4) \otimes \chi_{p}^{2} .
$$

Our main theorem expresses the right-hand side of (1.3) in terms of generating functions for explicit divisor sums. For this, define

$$
\mathcal{G}_{N, r}(q):=\sum_{n \geq 1} \sum_{\substack{d d^{\prime}=n \\ d \equiv \pm r \\(\bmod N) \\ d^{\prime}>d}} d q^{n}+\sum_{n \geq 1}(N n-r) q^{(N n-r)^{2}}
$$

Moreover, we let $S_{N, r}$ denote the operator $f \mid S_{N, r}(\tau)=\sum_{n \in \mathbb{Z}} a(N n+r) q^{N n+r}$ which sieves coefficients congruent to $r$ modulo $N$.

Theorem 1.4. For every $a \in \mathbb{Z}$ and odd prime $p$, we have that

$$
\left(\mathcal{H}(q) \vartheta_{a, p}(\tau)\right)\left|U(4) \otimes \chi_{p}^{2}+\sum_{\substack{b \\ b \neq \pm a}} \mathcal{G}_{p, a+b}(q)\right| S_{p, a^{2}-b^{2}}
$$

is a weight 2 holomorphic modular form on $\Gamma_{0}\left(p^{2}\right) \cap \Gamma_{1}(p)$. Moreover, if $a=0$, then (1.4) is a weight 2 modular form on $\Gamma_{0}\left(p^{2}\right)$.

This paper is organized as follows. In Section 2, we recall Hirzebruch and Zagier's completion of the class number generating function and introduce important series known as Appell-Lerch sums. In Section 3, we show how to complete the functions on either side of (1.4) to obtain non-holomorphic modular forms. We conclude the paper by proving Theorem 1.4 and Theorem 1.3 in Section 4 .

\section{Hurwitz Class numbers, Lerch sums, AND KNOWN FACTS}

As mentioned in the introduction, the generating function (1.1) for the Hurwitz class numbers is a mock modular form. More precisely, Hirzebruch and Zagier [9] proved 
that one can complete $\mathcal{H}$ by adding a certain simple sum involving the incomplete Gamma function

$$
\Gamma(s ; y):=\int_{y}^{\infty} t^{s-1} e^{-t} d t .
$$

The completed function is non-holomorphic, but belongs to a special class of functions known as harmonic weak Maass forms. To define these, we require the weight $k$ hyperbolic Laplacian (denoting $\tau=x+i y$ with $x, y \in \mathbb{R}$ throughout)

$$
\Delta_{k}:=-y^{2}\left(\frac{\partial^{2}}{\partial x^{2}}+\frac{\partial^{2}}{\partial y^{2}}\right)+i k y\left(\frac{\partial}{\partial x}+i \frac{\partial}{\partial y}\right)
$$

Weight $k$ harmonic weak Maass forms for $\Gamma \subset \mathrm{SL}_{2}(\mathbb{Z})$ are real analytic functions $\mathcal{F}: \mathbb{H} \rightarrow \mathbb{C}$ satisfying the following properties:

(1) $\left.\mathcal{F}\right|_{k} \gamma(\tau)=\mathcal{F}(\tau)$ for every $\gamma \in \Gamma$,

(2) $\Delta_{k}(\mathcal{F})=0$

(3) $\mathcal{F}$ has at most linear exponential growth at each cusp of $\Gamma$.

Here $\left.\right|_{k}$ is the usual weight $k$ slash operator. The class number generating function also belongs to a distinguished subspace, consisting of those forms whose $n$th coefficient (in the Fourier expansion in $x$ ) vanishes unless $(-1)^{k-\frac{1}{2}} n \equiv 0,1(\bmod 4)$, known as Kohnen's plus space [10].

We collect the modularity properties of $\mathcal{H}$ in the following theorem which can be easily concluded from Theorem 2 of [9].

Theorem 2.1. The function

$$
\widehat{\mathcal{H}}(\tau):=\mathcal{H}(q)+\frac{1}{4 \sqrt{\pi}} \sum_{n>0} n \Gamma\left(-\frac{1}{2} ; 4 \pi n^{2} y\right) q^{-n^{2}}+\frac{1}{8 \pi \sqrt{y}}
$$

is a weight $\frac{3}{2}$ harmonic weak Maass form on $\Gamma_{0}(4)$ in Kohnen's plus space.

A number of other completions help us to prove Theorem 1.4. To this end, for $u \in \mathbb{C} \backslash(\mathbb{Z} \tau+\mathbb{Z})$ we define the multivariable Appell function [15, 18]

$$
A_{\ell}(u, v ; \tau):=e^{\pi i \ell u} \sum_{n \in \mathbb{Z}} \frac{(-1)^{\ell n} q^{\frac{\ell}{2} n(n+1)} e^{2 \pi i n v}}{1-e^{2 \pi i u} q^{n}} .
$$

Directly from the definition, we have

$$
\begin{aligned}
& (-1)^{\ell} A_{\ell}(u+1, v ; \tau)=A_{\ell}(u, v+1 ; \tau)=A_{\ell}(u, v ; \tau) \\
& A_{\ell}(u+\tau, v+\ell \tau ; \tau)=(-1)^{\ell} q^{-\frac{\ell}{2}} e^{2 \pi i v} A_{\ell}(u, v ; \tau)
\end{aligned}
$$


In order to add a non-holomorphic function which "completes" $A_{\ell}$ to satisfy modularity, we define

$$
\begin{aligned}
\vartheta(z ; \tau) & :=\sum_{n \in \frac{1}{2}+\mathbb{Z}} e^{\pi i n^{2} \tau+2 \pi i n\left(z+\frac{1}{2}\right)} \\
R(u ; \tau) & :=\sum_{n \in \frac{1}{2}+\mathbb{Z}}\left(\operatorname{sgn}(n)-E\left(\left(n+\frac{\operatorname{Im}(u)}{y}\right) \sqrt{2 y}\right)\right)(-1)^{n-\frac{1}{2}} e^{-\pi i n^{2} \tau-2 \pi i n u}, \\
E(z) & :=2 \int_{0}^{z} e^{-\pi t^{2}} d t .
\end{aligned}
$$

We note that for $u \neq 0$, we also have the useful formula

$$
E(u)=\operatorname{sgn}(u)\left(1-\frac{e^{-\pi u^{2}}}{\pi|u|}+\frac{1}{2 \sqrt{\pi}} \Gamma\left(-\frac{1}{2} ; \pi u^{2}\right)\right) .
$$

Theorem 2.2 of [18] yields the transformation properties of the completion of $A_{\ell}$ :

$$
\begin{aligned}
\widehat{A}_{\ell}(u, v ; \tau) & :=A_{\ell}(u, v ; \tau) \\
& +\frac{i}{2} \sum_{k=0}^{\ell-1} e^{2 \pi i k u} \vartheta\left(v+k \tau+\frac{\ell-1}{2} ; \ell \tau\right) R\left(\ell u-v-k \tau-\frac{\ell-1}{2} ; \ell \tau\right) .
\end{aligned}
$$

Theorem 2.2. The function $\widehat{A}_{\ell}$ satisfies

$$
\widehat{A}_{\ell}\left(\frac{u}{c \tau+d}, \frac{v}{c \tau+d} ; \frac{a \tau+b}{c \tau+d}\right)=(c \tau+d) e^{\frac{\pi i c}{c \tau+d}\left(-\ell u^{2}+2 u v\right)} \widehat{A}_{\ell}(u, v ; \tau) .
$$

Moreover, for every $m_{1}, m_{2}, n_{1}, n_{2} \in \mathbb{Z}$, we have

$$
\begin{aligned}
\widehat{A}_{\ell}\left(u+n_{1} \tau+m_{1}, v+\right. & \left.n_{2} \tau+m_{2} ; \tau\right) \\
& =(-1)^{\ell\left(n_{1}+m_{1}\right)} e^{2 \pi i u\left(\ell n_{1}-n_{2}\right)} e^{-2 \pi i n_{1} v} q^{\frac{\ell}{2} n_{1}^{2}-n_{1} n_{2}} \widehat{A}_{\ell}(u, v ; \tau) .
\end{aligned}
$$

\section{Mixed MOCK MODUlAR FORMS AND NON-HOLOMORPHIC COMPLETIONS}

In this section, we show how to add a non-holomorphic function to "complete" each of the functions defined in the introduction to obtain a non-holomorphic modular

form. In order to write down these completions, for $N \in \mathbb{N}$ it is helpful to define the function

$$
R_{a, N}(\tau):=\sum_{\substack{n \in \mathbb{Z} \\ n \equiv a}}|n| \Gamma\left(-\frac{1}{2} ; 4 \pi n^{2} y\right) q^{-n^{2}} .
$$


Note that $R_{a, N}$ only depends on $a(\bmod N)$ and

$$
R_{-a, N}=R_{a, N}
$$

We further define the unary theta functions

$$
\vartheta_{a, N}(\tau):=\sum_{\substack{m \in \mathbb{Z} \\ m \equiv a}} q^{m^{2}} .
$$

3.1. In this subsection, we complete $\left(\mathcal{H}(q) \vartheta_{2 a, p}(\tau)\right) \mid U(4) \otimes \chi_{p}^{2}$.

Lemma 3.1. For every $a, b \in \mathbb{Z}$ and odd prime $p$, the function

$$
\begin{array}{r}
\left(\mathcal{H}(q) \vartheta_{2 a, p}(\tau)\right) \mid U(4) \otimes \chi_{p}^{2}+\frac{1}{8 \sqrt{\pi}} \sum_{\substack{b \\
b \neq \pm a(\bmod p)}} \sum_{k=0}^{1} R_{2 b+k p, 2 p}\left(\frac{\tau}{4}\right) \vartheta_{2 a+k p, 2 p}\left(\frac{\tau}{4}\right) \\
+\frac{\vartheta_{2 a, 2 p}\left(\frac{\tau}{4}\right)}{4 \pi \sqrt{y}}
\end{array}
$$

satisfies weight 2 modularity for $\Gamma:=\Gamma_{0}\left(p^{2}\right) \cap \Gamma_{1}(p)$.

Proof. By Proposition 2.1 of [16], $\vartheta_{2 a, p}$ is modular of weight $\frac{1}{2}$ on $\Gamma_{0}\left(4 p^{2}\right) \cap \Gamma_{1}(p)$.

We first write

$$
\left(\mathcal{H}(q) \vartheta_{2 a, p}(\tau)\right)\left|U(4) \otimes \chi_{p}^{2}=\sum_{1 \leq r \leq p-1}\left(\mathcal{H}(q) \vartheta_{2 a, p}(\tau)\right)\right| U(4) \mid S_{p, r}
$$

By Lemma 1 of [11], $\left(\widehat{\mathcal{H}}(q) \vartheta_{2 a, p}(\tau)\right) \mid U(2)$ fulfills weight 2 modularity on $\Gamma_{0}\left(2 p^{2}\right) \cap$ $\Gamma_{1}(2 p)$. Since the $n$th coefficient of $\left(\widehat{\mathcal{H}}(q) \vartheta_{2 a, p}(\tau)\right) \mid U(2)$ is zero unless $n$ is even, Lemma 4 of [1] implies that $\left(\widehat{\mathcal{H}}(q) \vartheta_{2 a, p}(\tau)\right) \mid U(4)$ satisfies weight 2 modularity on $\Gamma_{0}\left(p^{2}\right) \cap$ $\Gamma_{1}(p)$. Moreover, since modular forms on $\Gamma_{1}(p)$ split into modular forms on $\Gamma_{0}(p)$ with Nebentypus, rewriting $\chi_{p}^{2}=1-U(p) V(p)$ (where as usual $f \mid V(d)(\tau):=f(d \tau)$ ), Lemma 1 of [11] implies that the level goes down with $U(p)$ and back up with $V(p)$, so that overall the group becomes $\Gamma$.

By Theorem 2.1, to complete each summand $\left(\mathcal{H}(q) \vartheta_{2 a, p}(\tau)\right)|U(4)| S_{p, r}$ on the righthand side of (3.3), one must add

$$
\left(\frac{1}{4 \sqrt{\pi}} \sum_{\substack{n>0 \\ m \equiv 2 a}} n \Gamma\left(-\frac{1}{2} ; 4 \pi n^{2} y\right) q^{m^{2}-n^{2}}+\frac{1}{8 \pi \sqrt{y}} \sum_{m \equiv 2 a} q_{(\bmod p)}^{m^{2}}\right)|U(4)| S_{p, r} .
$$


Due to $m \equiv 2 a(\bmod p)$ and the congruences implied by $U(4)$ and $S_{p, r}$, the congruence conditions on $n$ and $m$ are equivalent to $m \equiv n(\bmod 2), m \equiv 2 a(\bmod p)$, and $n \equiv \pm 2 b(\bmod p)$, where $b$ satisfies $a^{2}-b^{2} \equiv r(\bmod p)$. If no such $b$ exists, then (3.4) equals zero. We may thus assume that such a $b$ exists. Whenever $b \not \equiv 0(\bmod p)$, the fact that $p$ is odd implies that (3.4) equals

$$
\frac{1}{8 \sqrt{\pi}}\left(\sum_{k=0}^{1} \sum_{ \pm} R_{ \pm 2 b+k p, 2 p}\left(\frac{\tau}{4}\right) \vartheta_{2 a+k p, 2 p}\left(\frac{\tau}{4}\right)\right) .
$$

Moreover, in the case that $b \equiv 0(\bmod p),(3.4)$ equals

$$
\frac{1}{8 \sqrt{\pi}}\left(\sum_{k=0}^{1} R_{k p, 2 p}\left(\frac{\tau}{4}\right) \vartheta_{2 a+k p, 2 p}\left(\frac{\tau}{4}\right)\right)+\frac{\vartheta_{2 a, 2 p}\left(\frac{\tau}{4}\right)}{4 \pi \sqrt{y}} .
$$

To finish the proof, one then sums over all choices of $b$ to obtain (3.2).

3.2. In this section, we complete $\sum_{ \pm} \mathcal{G}_{p, a \pm b}(q) \mid S_{p, a^{2}-b^{2}}$.

We begin with a lemma, which gives a more useful form $(\ell \in \mathbb{Z})$ for

$$
\mathcal{R}_{\ell, p}(v ; \tau):=\left[\frac{d}{d v}\left(e^{\frac{\pi i \ell v}{p}} q^{-\frac{\ell^{2}}{4}} R\left(p \ell \tau-v-\frac{1}{2} ; 2 p^{2} \tau\right)\right)\right]_{v=0} .
$$

Lemma 3.2. If $p$ is an odd prime and $\ell \in \mathbb{Z}$ satisfies $-p<\ell \leq p$, then

$$
\mathcal{R}_{\ell, p}(v ; \tau)=\sqrt{\pi} \sum_{n \in \frac{1}{2}+\mathbb{Z}}\left|n+\frac{\ell}{2 p}\right| \Gamma\left(-\frac{1}{2} ; 4 \pi p^{2}\left(n+\frac{\ell}{2 p}\right)^{2} y\right) q^{-p^{2}\left(n+\frac{\ell}{2 p}\right)^{2}}+\frac{\delta_{\ell=p}}{p \sqrt{y}} .
$$

Proof. We have

$$
\begin{aligned}
& e^{\frac{\pi i \ell v}{p}} q^{-\frac{\ell^{2}}{4}} R\left(p \ell \tau-v-\frac{1}{2} ; 2 p^{2} \tau\right) \\
& \quad=i \sum_{n \in \frac{1}{2}+\mathbb{Z}}\left(\operatorname{sgn}(n)-E\left(2 p\left(n+\frac{\ell}{2 p}-\frac{\operatorname{Im}(v)}{2 p^{2} y}\right) \sqrt{y}\right)\right) q^{-p^{2}\left(n+\frac{\ell}{2 p}\right)^{2}} e^{2 \pi i v\left(n+\frac{\ell}{2 p}\right)} .
\end{aligned}
$$

We now note that whenever $n+\frac{\ell}{2 p} \neq 0$, we have

$$
\operatorname{sgn}(n)=\operatorname{sgn}\left(n+\frac{\ell}{2 p}\right) .
$$

Differentiating and plugging in (2.4) yields the claim, after a straightforward calculation. 
In order to complete the right-hand side of (1.4), we pair the terms $b$ and $-b$ and determine the associated completion.

Lemma 3.3. Suppose that $a, b \in \mathbb{Z}$ and $p$ is an odd prime with $a \not \equiv \pm b(\bmod p)$.

(1) If $b \not \equiv 0(\bmod p)$, then the function

$$
\widehat{\mathcal{G}}_{p, a, b}(q):=\sum_{ \pm} \mathcal{G}_{p, a \pm b}(q) \mid S_{p, a^{2}-b^{2}}-\frac{1}{4 \sqrt{\pi}} \sum_{k=0}^{1} R_{k p+2 b, 2 p}\left(\frac{\tau}{4}\right) \vartheta_{k p+2 a, 2 p}\left(\frac{\tau}{4}\right)
$$

satisfies weight 2 modularity for $\Gamma$.

(2) If $b \equiv 0(\bmod p)$, then the function

$$
\widehat{\mathcal{G}}_{p, a, 0}(q):=\mathcal{G}_{p, a}(q) \mid S_{p, a^{2}}-\frac{1}{8 \sqrt{\pi}} \sum_{k=0}^{1} R_{k p, 2 p}\left(\frac{\tau}{4}\right) \vartheta_{k p+2 a, 2 p}\left(\frac{\tau}{4}\right)-\frac{\vartheta_{2 a, 2 p}\left(\frac{\tau}{4}\right)}{4 \pi \sqrt{y}}
$$

satisfies weight 2 modularity for $\Gamma$.

(3) In the case that $a \equiv 0(\bmod p)$, the function

$$
\sum_{0<b<\frac{p}{2}} \widehat{\mathcal{G}}_{p, 0, b} \mid S_{p,-b^{2}}
$$

furthermore satisfies weight 2 modularity for $\Gamma_{0}\left(p^{2}\right)$.

Proof. We first assume that $b \not \equiv 0(\bmod p)$ and may assume without loss of generality that $a+b<p$, since the definition of $\mathcal{G}_{p, a+b}$ only depends on $a$ and $b$ modulo $p$. Recalling the definition (2.2) of $A_{2}$, we obtain

$$
\begin{aligned}
& \mathcal{G}_{p, a+b}(q) \mid S_{p, a^{2}-b^{2}}=\sum_{n \equiv a^{2}-b^{2}} \sum_{(\bmod p)} \sum_{\begin{array}{c}
d d^{\prime}=n \\
d \equiv \pm(a+b) \\
d^{\prime}>d
\end{array}(\bmod p)} d q^{n} \\
& =\sum_{\substack{n \geq 0 \\
m \geq 0}}(p n+(a+b)) q^{(p n+(a+b))(p n+p+(a-b)+p m)} \\
& +\sum_{\substack{n \geq 1 \\
m \geq 0}}(p n-(a+b)) q^{(p n-(a+b))(p n+(b-a)+p m)} \\
& =\sum_{n \in \mathbb{Z}} \frac{(p n+(a+b)) q^{(p n+(a+b))(p n+p+(a-b))}}{1-q^{p(p n+(a+b))}} \\
& =\frac{p}{2 \pi i}\left[\frac{d}{d v}\left(A_{2}\left((a+b) p \tau, v+2 a p \tau ; p^{2} \tau\right) e^{\frac{2 \pi i(a+b) v}{p}} q^{a^{2}-b^{2}}\right)\right]_{v=0} .
\end{aligned}
$$


The analogous calculation for $-b$ follows by (2.3). A similar calculation yields a uniform equation for $b \equiv 0(\bmod p)$ and $a \not \equiv 0(\bmod p)$ as well. Overall, we obtain

$$
\mathcal{G}_{p, a \pm b}(q) \mid S_{p, a^{2}-b^{2}}=\frac{p}{2 \pi i}\left[\frac{d}{d v}\left(A_{2}\left((b \pm a) p \tau, v \pm 2 a p \tau ; p^{2} \tau\right) e^{\frac{2 \pi i(b \pm a) v}{p}} q^{a^{2}-b^{2}}\right)\right]_{v=0}
$$

We next prove the modularity of

$$
\widehat{G}_{b}(\tau):=\frac{p}{2 \pi i}\left[\frac{d}{d v}\left(\widehat{A}_{2}\left((a+b) p \tau, v+2 a p \tau ; p^{2} \tau\right) e^{\frac{2 \pi i(a+b) v}{p}} q^{a^{2}-b^{2}}\right)\right]_{v=0} .
$$

For $\left(\begin{array}{ll}\alpha & \beta \\ \gamma & \delta\end{array}\right) \in \Gamma$, we use equation (2.6) followed by (2.7) with $m_{1}=(a+b) \beta p, m_{2}=a \beta p$, $n_{1}=(b+a)\left(\frac{\alpha-1}{p}\right) \in \mathbb{Z}$, and $n_{2}=2 a\left(\frac{\alpha-1}{p}\right) \in \mathbb{Z}$ to yield

$$
\widehat{G}_{b}\left(\frac{\alpha \tau+\beta}{\gamma \tau+\delta}\right)=(\gamma \tau+\delta)^{2} \widehat{G}_{b}(\tau)
$$

Hence $\widehat{G}_{b}$ satisfies weight 2 modularity.

It remains to compute the non-holomorphic part of $\sum_{ \pm} \widehat{G}_{ \pm b}$. By (2.5) and the definition (3.5) of $\widehat{G}_{b}$, we have

$$
\begin{aligned}
\sum_{ \pm} \widehat{G}_{ \pm b}(\tau)= & \sum_{ \pm} \mathcal{G}_{p, a \pm b}(q) \mid S_{p, a^{2}-b^{2}}+\frac{p}{4 \pi}\left[\frac{d}{d v} \sum_{ \pm} \sum_{k=0}^{1} \vartheta\left(v \pm 2 a p \tau+k p^{2} \tau+\frac{1}{2} ; 2 p^{2} \tau\right)\right. \\
& \left.\times R\left(2 b p \tau-v-k p^{2} \tau-\frac{1}{2} ; 2 p^{2} \tau\right) e^{2 \pi i\left(a^{2}-b^{2}+k(b \pm a) p\right) \tau} e^{\frac{2 \pi i(b \pm a) v}{p}}\right]_{v=0}
\end{aligned}
$$

Here we double count $b \equiv 0(\bmod p)$ to get a uniform formula. We now rewrite

$$
\begin{aligned}
q^{\frac{1}{4}(k p \pm 2 a)^{2}} e^{\frac{2 \pi i v}{p}\left(\frac{k p}{2} \pm 2 a\right)} \vartheta\left(v \pm 2 a p \tau+k p^{2} \tau\right. & \left.+\frac{1}{2} ; 2 p^{2} \tau\right) \\
& =-\sum_{m \in \frac{1}{2}+\mathbb{Z}} q^{\left(m p+\frac{k p}{2} \pm a\right)^{2}} e^{\frac{2 \pi i v}{p}\left(m p+\frac{k p}{2} \pm a\right)}
\end{aligned}
$$


However, we have that

$$
\begin{aligned}
& \sum_{ \pm}\left[\frac{d}{d v} \sum_{m \in \frac{1}{2}+\mathbb{Z}} q^{\left(m p+\frac{k p}{2} \pm a\right)^{2}} e^{\frac{2 \pi i v}{p}\left(m p+\frac{k p}{2} \pm a\right)}\right]_{v=0} \\
& =\frac{2 \pi i}{p} \sum_{ \pm} \sum_{m \in \frac{1}{2}+\mathbb{Z}}\left(m p+\frac{k p}{2} \pm a\right) q^{\left(m p+\frac{k p}{2} \pm a\right)^{2}}=0,
\end{aligned}
$$

which can be seen by making the change of variables $m \rightarrow-m-k$. Moreover, by taking $m \rightarrow-m-k$, we also see that

$$
\sum_{ \pm} \sum_{m \in \frac{1}{2}+\mathbb{Z}} q^{\left(m p+\frac{k p}{2} \pm a\right)^{2}}=2 \sum_{m \in \frac{1}{2}+\mathbb{Z}} q^{\frac{1}{4}(2 m p+k p+2 a)^{2}}=2 \vartheta_{(1-k) p+2 a, 2 p}\left(\frac{\tau}{4}\right) .
$$

It follows that (3.6) equals

$$
-\frac{p}{2 \pi} \sum_{k=0}^{1} \mathcal{R}_{2 b-k p, p}(v ; \tau) \vartheta_{(1-k) p+2 a, 2 p}\left(\frac{\tau}{4}\right) .
$$

By Lemma 3.2, we may rewrite this as

$$
\begin{aligned}
&-\delta_{b=p} \frac{\vartheta_{2 a, 2 p}\left(\frac{\tau}{4}\right)}{2 \pi \sqrt{y}}-\frac{1}{4 \sqrt{\pi}} \sum_{k=0}^{1} \vartheta_{(1-k) p+2 a, 2 p}\left(\frac{\tau}{4}\right) \\
& \times \sum_{n \in \frac{1}{2}+\mathbb{Z}}|2 n p-k p+2 b| \Gamma\left(-\frac{1}{2} ; \pi(2 n p-k p+2 b)^{2} y\right) q^{-\frac{1}{4}(2 n p-k p+2 b)^{2}} \\
& \quad=-\frac{1}{4 \sqrt{\pi}} \sum_{k=0}^{1} R_{(1-k) p+2 b, 2 p}\left(\frac{\tau}{4}\right) \vartheta_{(1-k) p+2 a, 2 p}\left(\frac{\tau}{4}\right)-\delta_{b=p} \frac{\vartheta_{2 a, 2 p}\left(\frac{\tau}{4}\right)}{2 \pi \sqrt{y}} .
\end{aligned}
$$

Statements (1) and (2) now follow from Lemma 3.2 with $\ell=2 b-k p$.

We next prove part (3). The claim is equivalent to showing that

$$
g(\tau):=\frac{4 \pi i}{p} \sum_{0<b<\frac{p}{2}} \widehat{\mathcal{G}}_{p, 0, b} \mid S_{p,-b^{2}}=\left[\frac{d}{d v} \sum_{b} e_{(\bmod p)^{*}} e^{\frac{2 \pi i b v}{p}} q^{-b^{2}} \widehat{A}_{2}\left(b p \tau, v ; p^{2} \tau\right)\right]_{v=0}
$$

satisfies weight 2 modularity for $\Gamma_{0}\left(p^{2}\right)$. Here the sum runs over those $b(\bmod p)$ with $(b, p)=1$. Note that the sum only depends on $b(\bmod p)$ because, for $b^{\prime} \equiv b(\bmod p)$, 
we may use (2.7) with $n_{1}=\frac{b^{\prime}-b}{p}$. However, a simple calculation yields

$$
\begin{aligned}
g\left(\frac{\alpha \tau+\beta}{\gamma \tau+\delta}\right)=(\gamma \tau+\delta)^{2}\left[\frac{d}{d v} \sum_{b} q_{(\bmod p)^{*}} q^{-\alpha^{2} b^{2}} e^{\frac{2 \pi i b \alpha v}{p}} \widehat{A}_{2}\left(b \alpha p \tau, v ; p^{2} \tau\right)\right]_{v=0} & =(\gamma \tau+\delta)^{2} g(\tau)
\end{aligned}
$$

where we used that $b \alpha$ runs $(\bmod p)^{*}$ if $b$ does.

\section{Proof of Theorem 1.4 and Theorem 1.3}

In this section, we prove our main theorem and then give explicit identities for

$$
\sum_{\substack{n \geq 0 \\ p \nmid n}} H_{a, p}(n) q^{n}
$$

for certain fixed choices of $a$ and $p$. In particular, since the divisor sums occurring in Theorem 1.4 are particularly simple for primes, we obtain the desired conjectures leading to Theorem 1.3. We begin with the proof of our main theorem.

Proof of Theorem 1.4. By fixing $a$ and summing over all congruence classes for $b \not \equiv$ $\pm a(\bmod p)$ in Lemma 3.3, we see that the non-holomorphic parts cancel the nonholomorphic part from Lemma 3.1 and hence the sum is a (weakly) holomorphic modular form. Every coefficient of our new overall function may be written as a linear combination of class numbers and divisor sums and hence grows polynomially. Thus we have a holomorphic modular form of weight 2, yielding Theorem 1.4.

To compute explicit identities, we use the following lemma, which follows from the valence formula.

Lemma 4.1. If $p$ is an odd prime, $a \in \mathbb{Z}$, and $f$ is a holomorphic modular form of weight 2 on $\Gamma$, then

$$
\left(\mathcal{H}(q) \vartheta_{a, p}(\tau)\right)\left|U(4) \otimes \chi_{p}^{2}+\sum_{\substack{b \\ b \neq \pm a}} \mathcal{G}_{p, a+b}(q)\right| S_{p, a^{2}-b^{2}}=f(\tau)
$$

if and only if the first $\frac{p}{6}\left(p^{2}-1\right)$ Fourier coefficients agree.

Moreover, if $a \equiv 0(\bmod p)$ and $f$ satisfies weight 2 modularity for $\Gamma_{0}\left(p^{2}\right)$, then the above identity holds if and only if it holds for the first $\frac{p}{6}(p+1)$ coefficients. 
Denoting $\sigma(n):=\sum_{d \mid n} d$, we write the Eisenstein series part of the modular forms from Lemma 4.1 (in the special cases $p=3,5,7$ ) in terms of

$$
\mathcal{D}(q):=\sum_{n=1}^{\infty} \sigma(n) q^{n} .
$$

Since $\mathcal{D}$ is essentially a constant multiple of the weight 2 Eisenstein series $E_{2}$, it is well-known that

$$
\widehat{\mathcal{D}}(\tau):=\mathcal{D}(q)-\frac{1}{24}+\frac{1}{8 \pi y}
$$

transforms like a modular form of weight 2 on $\mathrm{SL}_{2}(\mathbb{Z})$. In particular, since every nontrivial character $\chi$ satisfies $\chi(0)=0$, the function $\mathcal{D} \otimes \chi$ is a weight 2 holomorphic modular form. More precisely, if the modulus of $\chi$ is $m \in \mathbb{N}$, then $\mathcal{D} \otimes \chi$ is a weight 2 holomorphic modular form on $\Gamma_{0}\left(m^{2}\right)$ (cf. Proposition 2.8 of [13]). Furthermore, if $r \not \equiv 0(\bmod p)$, then a straightforward calculation shows that $\mathcal{D} \mid S_{p, r}$ is a holomorphic modular form of weight 2 on $\Gamma$.

It is well-known (cf. Section 7.2, Example 2 in [7]) that

$$
\left(\mathcal{H} \vartheta_{0,1}\right) \mid U(4)=2 \mathcal{D}-\mathcal{G}_{1,0}-\frac{1}{12}
$$

while one sees directly that $\vartheta_{0,1}=\sum_{a(\bmod p)} \vartheta_{a, p}$ and $\vartheta_{a, p}=\vartheta_{-a, p}$. Hence we only need to determine formulas for the modular forms from Lemma 4.1 whenever $0 \leq a \leq \frac{p-3}{2}$ to obtain them for all $a \in \mathbb{Z}$. After constructing such modular forms, we use (1.3) to conclude Theorem 1.3. For simplicity, we only work out the exact identities for $a=0$.

4.1. $p=3$. We include the case $p=3$ since the formulas are particularly simple in this case and because it indicates the general method well. Using the fact that $\mathcal{D} \otimes \chi_{3}$ is a holomorphic modular form of weight 2 for $\Gamma_{0}(9)$, Lemma 4.1 implies that

$$
\left(\mathcal{H} \vartheta_{0,3}\right)\left|U(4) \otimes \chi_{3}^{2}=-2 \mathcal{G}_{3,1}\right| S_{3,2}+\mathcal{D} \otimes \chi_{3}^{2}-\frac{1}{4} \mathcal{D} \otimes \chi_{3}\left(1+\chi_{3}\right) .
$$

The $n$th coefficient of $-2 \mathcal{G}_{3,1} \mid S_{3,2}$ is 0 unless $n \equiv 2(\bmod 3)$, in which case it equals

$$
-2 \sum_{\substack{d \equiv \pm 1 \\ d \mid n, d<\frac{n}{d}}} d .
$$

The $n$th coefficient of $\mathcal{D} \otimes \chi_{3}^{2}$ is 0 if $3 \mid n$ and otherwise $\sigma(n)$. Finally, the $n$th coefficient of $-\frac{1}{4} \mathcal{D} \otimes \chi_{3}\left(1+\chi_{3}\right)$ is 0 unless $n \equiv 1(\bmod 3)$, in which case it equals $-\frac{1}{2} \sigma(n)$. Thus 
the overall $n$th coefficient on the right-hand side of (4.2) is

$$
\left\{\begin{array}{lll}
\frac{1}{2} \sigma(n) & \text { if } n \equiv 1 & (\bmod 3), \\
\sigma(n)-2 \sum_{\substack{d \equiv \pm 1 \\
d \mid n, d<\frac{n}{d}}} d & \text { if } n \equiv 2 & (\bmod 3) .
\end{array}\right.
$$

Comparing with (1.3), we get in particular for a prime $\ell>3$

$$
H_{0,3}(\ell)=\left\{\begin{array}{lll}
\frac{\ell+1}{2} & \text { if } \ell \equiv 1 \quad(\bmod 3), \\
\ell-1 & \text { if } \ell \equiv 2 \quad(\bmod 3) .
\end{array}\right.
$$

4.2. $p=5$. The precise version of Conjecture 1.1 in [6] is given by the following.

Conjecture 4.2. For a prime $\ell$ and $a \in \mathbb{Z}$ one has that

$$
H_{a, 5}(\ell)= \begin{cases}\frac{\ell+1}{2} & \text { if } a \equiv 0 \quad(\bmod 5), \text { and } \ell \equiv 1 \quad(\bmod 5), \\ \frac{\ell+1}{3} & \text { if } a \equiv 0 \quad(\bmod 5), \text { and } \ell \equiv 2,3 \quad(\bmod 5), \\ \frac{\ell+1}{3} & \text { if } a \equiv \pm 1 \quad(\bmod 5), \text { and } \ell \equiv 1,2 \quad(\bmod 5), \\ \frac{5 \ell+5}{12} & \text { if } a \equiv \pm 1 \quad(\bmod 5), \text { and } \ell \equiv 4 \quad(\bmod 5), \\ \frac{5 \ell-7}{12} & \text { if } a \equiv \pm 2 \quad(\bmod 5), \text { and } \ell \equiv 1 \quad(\bmod 5), \\ \frac{\ell+1}{3} & \text { if } a \equiv \pm 2 \quad(\bmod 5), \text { and } \ell \equiv 3,4 \quad(\bmod 5) .\end{cases}
$$

We prove Conjecture 4.2 by showing the following more precise version.

Corollary 4.3. One has that

$$
\begin{aligned}
\left(\mathcal{H} \vartheta_{0,5}\right) \mid U(4) \otimes \chi_{5}^{2} & =\frac{1}{2} \mathcal{D} \otimes \chi_{5}^{2}-\frac{1}{12} \mathcal{D} \otimes \chi_{5}\left(\chi_{5}-1\right)-2 \mathcal{G}_{5,1}\left|S_{5,4}-2 \mathcal{G}_{5,2}\right| S_{5,1} \\
\left(\mathcal{H} \vartheta_{1,5}\right) \mid U(4) \otimes \chi_{5}^{2}= & \frac{1}{3} \mathcal{D} \otimes \chi_{5}^{2}+\left(\frac{1}{6} \mathcal{D}-\mathcal{G}_{5,1}-\mathcal{G}_{5,2}\right) \mid S_{5,3} \\
& +\left(\frac{1}{12} \mathcal{D}-\frac{1}{2} \mathcal{G}_{5,2}-\frac{1}{2} \mathcal{G}_{5,3}\right) \mid S_{5,4}
\end{aligned}
$$

In particular, the conjectured formula (4.3) is true.

Proof. The holomorphic modular forms occurring by twisting $\mathcal{D}$ with a character of modulus 5 have level $\Gamma_{0}(25)$, while $\mathcal{D} \mid S_{5, a}(a \not \equiv 0(\bmod 5))$ is a holomorphic modular form for $\Gamma$. After checking 20 coefficients, Lemma 4.1 yields the equalities claimed in the corollary. To obtain (4.3), we simply apply an analysis similar to that used in the case for $p=3$ above to obtain the explicit coefficients. 
4.3. $p=7$. Conjecture 1.2 follows from the following more precise version.

Conjecture 4.4. For a prime $\ell$ and $a \in \mathbb{Z}$ one has that

$$
H_{a, 7}(\ell)=\left\{\begin{array}{llll}
\frac{\ell+1}{3} & \text { if } a \equiv \pm 1 & (\bmod 7), & \text { and } \ell \equiv 1 \quad(\bmod 7), \\
\frac{\ell+1}{4} & \text { if } a \equiv \pm 1 & (\bmod 7), & \text { and } \ell \equiv 3,6 \quad(\bmod 7) \\
\frac{\ell+1}{4} & \text { if } a \equiv \pm 2 & (\bmod 7), & \text { and } \ell \equiv 3,5 \quad(\bmod 7) \\
\frac{\ell+1}{4} & \text { if } a \equiv \pm 3 & (\bmod 7), & \text { and } \ell \equiv 5,6 \quad(\bmod 7),
\end{array}\right.
$$

The argument is analogous to the above cases for $p=3$ and $p=5$, except that there are cusp forms in the spaces of interest. For example, for $a=0$, we require the weight 2 newform (of level 49) $g_{7}$ associated to the elliptic curve $y^{2}+x y=x^{3}-x^{2}-2 x-1$ (which is denoted $49 A 1$ and has $\mathrm{CM}$ by $\mathbb{Q}(\sqrt{-7}))$. The coefficients of $g_{7}$ are multiplicative and Parry has explicitly written $g_{7}$ in terms of the two variable Ramanujan theta function.

After comparing the first 56 coefficients in the identity, Lemma 4.1 immediately yields the following corollary.

Corollary 4.5. One has that

$$
\begin{aligned}
\left(\mathcal{H} \vartheta_{0,7}\right) \mid U(4) \otimes \chi_{7}^{2}=\frac{1}{4} \mathcal{D} \otimes \chi_{7}^{2}+\frac{1}{24} & \mathcal{D} \otimes \chi_{7}\left(\chi_{7}-1\right) \\
& -2 \mathcal{G}_{7,2}\left|S_{7,3}-2 \mathcal{G}_{7,4}\right| S_{7,-2}-2 \mathcal{G}_{7,1} \mid S_{7,-1}+\frac{1}{4} g_{7} .
\end{aligned}
$$

We do not work out the details for the cusp forms for $a \not \equiv 0(\bmod 7)$, but rather list the resulting identity when restricting to coefficients in certain congruence classes where the coefficients of the cusp forms are all zero. This suffices to prove Conjecture 4.4 .

Corollary 4.6. The following identities hold.

(1) For $r \equiv 1,3,6(\bmod 7)$, one has

$$
\left(\mathcal{H} \vartheta_{1,7}\right)|U(4)| S_{7, r}=\frac{1}{4} \mathcal{D}+ \begin{cases}\frac{1}{12} \mathcal{D}-\mathcal{G}_{7,2}-\mathcal{G}_{7,3} & \text { if } r \equiv 1 \quad(\bmod 7), \\ 0 & \text { if } r \equiv 3,6 \quad(\bmod 7) .\end{cases}
$$

(2) For $r \equiv 3,5(\bmod 7)$, one has

$$
\left(\mathcal{H} \vartheta_{2,7}\right)|U(4)| S_{7, r}=\frac{1}{4} \mathcal{D} .
$$

(3) For $r \equiv 5,6(\bmod 7)$, one has

$$
\left(\mathcal{H} \vartheta_{3,7}\right)|U(4)| S_{7, r}=\frac{1}{4} \mathcal{D} .
$$


In particular, Conjecture 4.4 is true.

\section{REFERENCES}

[1] K. Bringmann and A. Folsom, Almost harmonic Maass forms and Kac Wakimoto characters, J. reine und angew. Math., accepted for publication.

[2] K. Bringmann and S. Murthy, On the positivity of black hole degeneracies in string theory, Commun. Number Theory Phys., recommended for publication.

[3] K. Bringmann and K. Ono, The $f(q)$ mock theta function conjecture and partition ranks, Invent. Math. 165 (2006), 243-266.

[4] K. Bringmann and K. Ono, Some characters of Kac and Wakimoto and nonholomorphic modular functions, Math. Ann. 345 (2009), 547-558.

[5] K. Bringmann and K. Ono, Dyson's rank and Maass forms, Ann. of Math. 171 (2010), 419-449.

[6] B. Brown, N. Calkin, T. Flowers, K. James, E. Smith, and A. Stout, Elliptic curves, modular forms, and sums of Hurwitz class numbers, J. Number Theory 128 (2008), 1847-1863.

[7] A. Dabholkar, S. Murthy, and D. Zagier, Quantum black holes, wall crossing and mock modular forms, submitted for publication.

[8] M. Eichler, On the class of imaginary quadratic fields and sums of divisors of natural numbers, J. Indian Math. Soc. 19 (1956), 153-180.

[9] F. Hirzebruch and D. Zagier, Intersection numbers of curves on Hilbert modular surfaces and modular forms of Nebentypus, Invent. Math. 36 (1976), 57-113.

[10] W. Kohnen, Modular forms of half-integral weight on $\Gamma_{0}(4)$, Math. Ann. 248 (1980), 249-266.

[11] W. Li, Newforms and functional equations, Math. Ann. 212 (1975), 285-315.

[12] K. Mahlburg, Partition congruences and the Andrews-Garval-Dyson crank, Proc. Natl. Acad. Sci. USA 102 (2005), 15373-15376.

[13] K. Ono, The web of modularity: arithmetic of the coefficients of modular forms and q-series, CBMS Regional Conference Series in Mathematics, 102. Published for the Conference Board of the Mathematical Sciences, Washington, DC; by the American Mathematical Society, Providence, RI, 2004.

[14] W. Parry, A negative result on the representation of modular forms by theta series, J. Reine Angew. Math. 310 (1979), 151-170.

[15] A. Semikhatov, A. Taormina, and I. Tipunin, Higher-level Appell functions, modular transformations, and characters, Comm. Math. Phys. 255 (2005), 469-512.

[16] G. Shimura, On modular forms of half integral weight, Ann. of Math. 97 (1973), 440-481.

[17] D. Zagier, Introduction to modular forms in "From Number Theory to Physics", Springer-Verlag, Heidelberg (1992), 238-291.

[18] S. Zwegers, Multivariable Appell functions, preprint.

[19] S. Zwegers, Mock theta functions, Ph.D. thesis, Utrecht University (2002).

Mathematical Institute, University of Cologne, Weyertal 86-90, 50931 Cologne, Germany

E-mail address: kbringma@math.uni-koeln.de

Department of Mathematics, University of Hong Kong, Pokfulam, Hong Kong E-mail address: bkane@maths.hku.hk 